\title{
The Brief Strategic Treatment of Cardiophobia: A Clinical Case Study
}

\author{
Giada Pietrabissa ${ }^{1,2}\left(\right.$ Federica Rozzoni $^{2} \cdot$ Flavia Liguori $^{3} \cdot$ Antonella Cerruto $^{3} \cdot$ Emanuele Maria Giusti $^{1,2}$. \\ Gabriella Malfatto $^{4}$ - Carlotta Munforti ${ }^{4} \cdot$ Martina De Martin $^{4}$. Alessia Giglio ${ }^{4} \cdot$ Mario Facchini $^{4}$. \\ Gianluca Castelnuovo ${ }^{1,2}$
}

Accepted: 10 November 2020 / Published online: 23 November 2020

(c) The Author(s) 2020

\begin{abstract}
Many individuals presenting to medical settings with heart-related symptoms for which no medical explanation is found might suffer from cardiophobia, but this condition is still poorly identified and addressed. This article presents a case of cardiophobia treated in an outpatient cardiac rehabilitation unit and, for the first time, describes the application of brief strategic therapy for the treatment of this condition. In the case reported, the first therapeutic encounter and the key elements of the strategic approach are described in detail with the aim to explain how brief strategic therapy works and how it can be used to identify and address cardiophobia-related behaviors. A 64-year-old male presented to cardiac rehabilitation reporting intense anxiety-provoking heart palpitations, and believing he was at risk of dying from a heart attack. After 3 sessions, an overall improvement in heart-related bodily sensations followed a decrease in the patient's continuous checking of his heartbeat and seeking reassurance-factors that were largely responsible for the persistence of the problem. Moreover, quantitative evaluation showed increased scores of mood state at the end of treatment. This improvement persisted at the 18-month follow-up. This case is an interesting example of how brief strategic therapy can contribute to the development of a new conceptual model for the diagnosis and treatment of cardiophobia. Still, more systematic research in the field is needed to prove the efficacy and effectiveness of this therapeutic approach on symptoms of heart-focused anxiety.
\end{abstract}

Keywords Heart-focused anxiety $\cdot$ Cardiophobia $\cdot$ Cardiovascular diseases $\cdot$ Cardiac rehabilitation $\cdot$ Brief strategic therapy $\cdot$ Clinical psychology

\section{Introduction}

A significant proportion of patients presenting to medical consultation with complaints of chest pain, heart palpitations, and dizziness-but an absence of any organic problem-might suffer from cardiophobia. Cardiophobia is an anxiety disorder characterized by excessive worry about heart-related sensations and other somatic symptoms,

Giada Pietrabissa

giada.pietrabissa@unicatt.it

1 Clinical Psychology Lab, Istituto Auxologico Italiano IRCCS, Milan, Italy

2 Department of Psychology, Catholic University of Milan, Milan, Italy

3 Brief Strategic Therapy Center, Arezzo, Italy

4 Cardiological Rehabilitation Unit, Department of Cardiovascular, Neurological, Metabolic Sciences, Istituto Auxologico Italiano IRCCS, San Luca Hospital, Milan, Italy accompanied by fear of having a heart attack and of dying (Tremblay et al. 2018). Persons with cardiophobia perform anxious heart monitoring when experiencing stress and arousal and-despite repeated negative medical tests-they continue to believe and to ruminate in an almost obsessive fashion that they suffer from an organic heart problem that is life-threatening. These subjects typically seek continuous reassurance and support from significant others, and avoid activities believed to elicit symptoms (Eifert et al. 1996). Recurrent chest pain, continued fear, and enduring preoccupation about the heart functioning not only cause personal discomfort, but also have devastating consequences on the individual's social and occupational life (Eifert et al. 2000). A study by Ockene et al. (1980) reported that 16 months after cardiac angiography, $51 \%$ of persons with normal results were still unable to work because of perceived chest pain, $47 \%$ described their activity as limited, and $44 \%$ continued to believe they were suffering from heart disease (Ockene et al. 1980). Similarly, Bass et al. (1983) found that 
more than $65 \%$ of patients with noncardiac chest pain complained of continuing discomfort for more than 6 years after the initial visit (Bass et al. 1983). Not surprisingly, the cost to the health care system is immense in terms of repeated unnecessary visits to doctors, hospitalizations, and continual laboratory tests (Aikens et al. 2001), and inappropriately prescribed cardiac medications (Beitman et al. 1991).

Unfortunately, cardiophobia is rarely identified or diagnosed in medical settings, particularly if chest pain is the primary presenting medical problem (Eifert 1992). Early detection of cardiophobic behaviors by medical staff would not only result in more timely interventions, but might also decrease health care costs (Ginsberg et al. 2015).

Among the psychological treatments available, a review of eight studies suggests cognitive-behavioral therapy has a modest to moderate benefit for patients with cardiophobia (Kisely et al. 2015). However, there is no clear knowledge of the mechanisms through which the cognitive- behavioral approach to mental health operates (Spinhoven et al. 2010).

Brief strategic therapy might overcome this gap by providing a comprehensive framework of how people create their problem, and of how the problem functions in terms of what maintains and feeds it (Nardone and Balbi 2008). Fundamental to this approach is the concept of attempted solutions, which are the reactions of a person who is confronting a given difficulty in relationship with the self, the others, and the world. These reactions often complicate rather than solve the problem and end up becoming rigid, redundant, dysfunctional models of interaction with reality (Nardone and Portelli 2005). In order to substitute the dysfunctional attempted solutions with more functional ones, it is necessary to study the mental, emotional, and relational "traps" in which people might find themselves. Then, since the only variable that a person can control is his/her strategy, in order, the strategic therapist actively intervenes to identify and solve a given problem by introducing a change in the individual's harmful behaviors (dysfunctional attempted solutions).

The way a person responds to these changes will reveal the functioning of the phenomenon. As a final effect, change may take place even on a cognitive level, but only once the pathology has been unblocked. The therapist, therefore, gets to know a problem through its solution (Nardone and Portelli 2005). This notion goes beyond traditional approaches in psychotherapy_-which look first for awareness to produce change-and allows the strategic therapist to create functional interventions tailored to each given situation, rather than based on an a priori theory or categorization of mental disorders. By doing so, the nosological dilemma concerning the controversial diagnosis of cardiophobia might therefore be overcome.

In operative terms, specific communication techniques and indirect stratagems are applied in order to circumvent the individual's resistance to change his/her behaviors. This leads the person to experience a corrective emotional experience, then to spontaneously and permanently change his /her reactions to reality (Nardone and Watzlawick 2004). Specifically, the strategic dialog is an advanced communication technique purposely structured to help the therapist understand the persistence of the problem, and simultaneously make the patient "feel" things differently. Questions are close-ended-maintaining an illusion of alternatives in a funnel-like sequence. The alternative answers refer to the patient's possible perceptions of the problem and to his attempted solutions; the evolution of the dialogue opens up a different scenario requiring diverse strategies. After every two to three questions, the content of the responses provided is paraphrased to ensure mutual understanding and to bring the failed attempted solutions to light. This technique was developed through a natural evolutionary process of specific protocols for different types of psychopathology: rigorous action-research revealed that each psychopathology is maintained by a series of specific redundant reactions of a person who is facing recurring difficulties (Nardone and Salvini 2007). A few experimental studies also provided support for the value of brief strategic therapy in increasing psychological outcomes in people with cardiovascular disease (Pietrabissa et al. 2015, 2017; Rakowska 2015). Specifically, a randomized clinical trial by Rakowska (2015) showed improved mental and physical health in patients with myocardial infarction at treatment termination and at 2.5year follow-up by adding brief strategic therapy to usual care (Cohen's $d=0.40$ ). Similarly, in a randomized, singleblind study aimed at testing the incremental efficacy of brief strategic therapy combined with motivational interviewing compared to stand-alone brief strategic treatment in enhancing selected biomedical and psychological outcomes in a residential cardiac rehabilitation, Pietrabissa et al. (2017) showed no significant between-group difference in any outcome variable, but significant positive changes in the level of the participants' self-regulation and willingness to change from pre- to post-treatment, as well as maintenance of the results at the 3-month follow-up (Cohen's $d=0.35$ ). Among phobic disorders, 10 weeks of brief strategic therapy resulted in a greater reduction in social phobia symptoms compared to minimal supportive therapy at treatment termination and at the 3-month follow-up (Rakowska 2011). Obsessive-compulsive and depressive symptoms also decreased (Cohen's $d=0.5$ ) in a longitudinal study with a 3-month follow-up (Pietrabissa et al. 2016). Lastly, brief strategic therapy was statistically and clinically superior to cognitive-behavioral therapy in improving binge eating frequency, weight, and global functioning in a sample of women enrolled in an 1-month inpatient nutritional rehabilitation program (Castelnuovo et al. 2011; Jackson et al. 2018).

This article presents a case of cardiophobia treated in an outpatient cardiac rehabilitation unit and, for the first time, 
describes the application of brief strategic therapy for the treatment of this condition. In the case reported, the first therapeutic encounter and the key elements of the strategic approach are described in detail with the aim to make clear to the reader how the therapy works and how it can be used in clinical settings to promptly identify the presence of cardiophobic behaviors in order to subvert their maintenance mechanisms. From this case, the reader can also see how, in this approach, the usual process of all psychotherapies is inverted: in fact, while the majority of therapies look first for awareness in order to produce change, in brief strategic therapy, awareness follows the achieved changes.

\section{Methods}

\section{Case Presentation}

At the time of consultation, Walter (pseudonym) was attending the second week of the outpatient cardiac rehabilitation program of the San Luca Hospital, IRCCS Istituto Auxologico Italiano, Milan, Italy_following a recent heart attack.

Walter was 64, his body mass index (BMI) fell within the healthy weight range $\left(\mathrm{BMI}=24.72 \mathrm{~kg} / \mathrm{m}^{2}\right)$. He was following a healthy, balanced diet prescribed by the hospital dietitian for treating his dyslipidemia. His level of daily physical activity, previously maintained, progressively diminished after the cardiac event as a precautionary measure. He denied alcohol use but admitted to smoking about 20 cigarettes per day up until a year before. He worked as a freelancer, he was married and had a teenage son. Walter expressed satisfaction with his job, family and marital relationships, and social life.

\section{Measures}

As part of the outpatient 3-month cardiac rehabilitation, the following psychological outcomes were collected at beginning and termination of the program through self-report measures:

The Psychological General Well-Being Index (PGWBS) (Grossi et al. 2006). The 6-item version of the original PGWB is used to assess the psychological and general well-being of a person in six health-related quality of life (HRQoL) domains: anxiety, depressed mood, positive wellbeing, self-control, general health and vitality. Items are summed to produce an overall score ranging from 0 to 30 . The PGWBI-S has revealed high Cronbach's alpha coefficients in both its original $(\alpha=0.94)$ (Dupuy 1984) and Italian version (0.80-0.92) (Grossi et al. 2006).

The visual analogue scale of the EuroQol-5D (EQ-5D) is a well-known generic measure of HRQoL with a grade ranging from 0 (the worst possible) to 100 (the best possible)
(EuroQol Group 1990). The EQ-5D revealed a good internal consistency $(\alpha=0.73)$ in an Italian sample of patients following cardiac rehabilitation (Balestroni and Bertolotti 2012).

The Patient Health Questionnaire-4 (PHQ-4), a 4-item inventory rated on a 4-point Likert-type scale derived by combining the first two questions of the Generalized Anxiety Disorder-7 scale (known as GAD-2) and of the PHQ-8 (known as PHQ-2). Total score ranges from 0 to 12 (anxiety subscale: score range, 0 to 6 ; depression subscale: score range, 0 to 6 ), and is determined by adding together the scores of each of the 4 items. Scores are rated as normal (0-2), mild (3-5), moderate (6-8), and severe (9-12). The PHQ-4 revealed a good internal reliability $(\alpha>0.80)$ (Kroenke et al. 2009).

The Morisky Medication Adherence 4-item Scale (MMAS-4) is a 4-item scale with a no/yes response option for each item to which a score of $0 / 1$ is assigned. Total possible score ranges from 0 (full compliance) to 4 (worst compliance). The reliability of the scale is reflected in its acceptable measure of internal consistency $(\alpha=0.61)$ (Morisky et al. 1986).

\section{Procedure}

The cardiac rehabilitation program includes 20 supervised and individualized sessions over 12 weeks, and is supported by a multidisciplinary team of cardiologists, exercise specialists, dietitians or nutritionists, and mental health professionals. Before starting the program, the rehabilitation team takes the medical history and carries out an extensive medical assessment of patients. Psychological evaluation takes place at the beginning and at the end of the rehabilitation program through administration of a battery of selected questionnaires. For those showing high levels of anxiety, depression, reduced health-related quality of life or dysfunctional lifestyles, psychological support is provided.

For Walter, psychological evaluation was requested by a cardiologist member of the rehabilitation staff because of his constant complaining and worrying about a perceived irregular heartbeat, with no associated, aggravating, or relieving medical factors detected.

Baseline quantitative evaluation of his mood state revealed mild anxiety-depressive symptomatology with a prevalence of anxiety symptom (PHQ-4, anxiety scale $=3$ ), as well as a modest perceived HRQoL (PGWB-S $=17$; EQ-5D $=40$ ), and a moderate adherence to medical recommendations (MMAS $=2$ ).

The patient was seen three times by the psychologist-a specialist in brief strategic therapy working in the cardiac rehabilitation unit—at 2-week intervals, as provided for in the hospital protocol. 
At the end of the rehabilitation program, the patient showed a decreased anxiety score (PHQ-4, anxiety scale $=2$ ), as well as improved HRQoL (PGWB-S $=18$; $\mathrm{EQ}-5 \mathrm{D}=90$ ), while his adherence to medication remained moderate $(\mathrm{MMAS}=2)$.

All procedures performed in the study were run in accordance with the ethical standards of the institutional and/or national research committee and with the Helsinki Declaration and its later amendments or comparable ethical standards. Patient informed consent was obtained at his admission to the hospital. Moreover, during the last follow-up phone call-18 months after the end of the rehabilitation program - the patient was also asked for prior permission to share adequately de-identified information within the scientific community.

\section{First Session}

The first person entering the room was Walter's wife, who introduced herself in her role as a professional nurse, and quickly announced the patient before leaving the room.

Walter seemed to be in a state of tension but still collaborative during the entire encounter.

The therapist started by investigating the patient's perspective about his clinical situation, emotional status, lifestyle habits, and occupational, domestic, and social life.

Walter demonstrated extensive knowledge of his cardiac disease. He also acknowledged a previous nervous breakdown treated with drugs and autogenic training, and current symptoms of anxiety partially controlled through the use of anxiolytic drugs, as needed.

The therapist proceeded by asking the patient to define the problem that brought him to consultation. Walter showed awareness of the fact that his perceived irregular heartbeat could have a psychological, rather than a medical nature. Still, this did not reassure him, but rather seemed to increase his already existing symptoms of anxiety.

Next, the strategic dialogue was used to investigate how the problem manifests and how Walter has been trying to cope with the situation (attempted solutions). The therapist started by asking Walter: "When you start feeling that your heartbeat in increasing, do you fear losing control or dying?" and the patient replied: "I am afraid to die from a heart attack!" In this way, the problem is already restricted within the phobic area. Walter also specified fearing his own body at a functional level, indicating the possible presence of pathophobia.

This condition is distinct from hypochondria because the subject does not complain about multiple symptoms, but is afraid of suddenly dying due to the malfunction of his heart, which is commonly found in persons with a cardiac problem (Marker et al. 2008).
Walter's unique fear for the random rising of his heart rate was, in fact, confirmed by the patient's answer to the following questions: "Does it [rapid heartbeat] happen in situations that you can predict, or is it unpredictable?" and "Are you worried only about the sound of your heartbeat rising or are there any other symptoms you detect that make you feel anxious?"

Then a paraphrase was use to ensure mutual agreement on the problem: "Please correct me if I'm wrong [taking up a one-down submissive position] ... but you are telling me that you are experiencing symptoms of anxiety, and this corresponds to a fear of dying from a heart attack due to the rise in your heart rate, which happens in situations that you cannot predict and that does not apparently have any medical meaning. It that right?"

By declaring "Please, correct me if I'm wrong", the patient feels understood, emotionally reinforced, and acknowledged. This also allows the professional to establish an emotionally positive therapeutic relationship.

Then, the therapist proceeded by investigating Walter's attempted solutions by asking him: “... And the fact that the medical examinations reveal that there is nothing to worry about, reassures you or worries you?" And "when you start feeling that your heartbeat is increasing, do you tend to avoid or to try to control it?"

As expected, Walter felt relieved about the negative result of the medical analyses, but his worry did not seem to diminish. Moreover, he constantly monitored his bodily signals in order to identify any irregularity and promptly intervene in it.

To start creating aversion toward this behavior-instead of using the descriptive language of the explanation-the therapist adopted an evocative language driven by metaphors: "You are just like a sort of broken marionette with its eyes turned inwards; always looking at what is happening on the inside". Then, she proceeded by asking the patient: "Does monitoring your heartbeat helps you to control it or not?". Walter couldn't help but affirm that this strategy not only had never helped him, but it made him feel even worse due to repeated failure in controlling/regulating his heartbeat.

Thus, the following question was: “... And when you can't control your heartbeat, what do you do: do you ask for help or do you face it on your own?" and-as generally happens-Walter replied he would ask for help exclusively from his wife who, as a nurse, promptly meets the requests of her husband by measuring his parameters, and by making sure he takes the correct medicines in the right doses, and at the right time.

This is a very important question since it helps to evaluate whether the person is dependent or relies on someone, and this will orient the therapist to a completely different evolution of the treatment. 
Persons with pathophobia—unlike those who suffer from hypochondria, who tend to ask for help from a broader audience-usually request assistance only from specialists and significant others.

In order to instill the doubt in the patient that even this strategy could not help him to solve the problem-and thus to indirectly guide him towards change- the therapist continued by saying: "Well, I am sure that when you speak about your problem or ask for help from your wife, in that moment you feel better because you feel reassured. But after a while, do you feel better or still worried about the problem?" Once again, Walter admitted to still feeling discouraged.

Then, a final paraphrase was used to confirm and redefine "Ok. Please allow me to recap what has been said and, please correct me if I'm wrong. You are a person who is afraid of dying from a heart attack due to the perceived irregularity of your heartbeat, which takes place in situations you cannot predict, and which you tend to control through medical screenings or by listening to your bodily signals. When you do so, you first feel relieved, but afterwards this makes you feel worse, as it does not help you to find an explanation or a solution to your problem. And not even asking for help or reassurance from your wife gives you relief in the long term, but later you feel even more unable to handle the situation on your own".

In virtue of these questions, the patient and the therapist together discovered how the problem functions based on three main dysfunctional attempted solutions. In fact, the patient's attempt to voluntarily control his symptoms by paying excessive attention to his body's signals has a paradoxical effect: the continuous listening to the heart's rhythm to diagnose promptly any potential disease led Walter to precisely find those signals that most frightened him. Consequently, the heart rate increased in response to the resulting distress. Similarly, asking for medical checks led to a series of negative diagnostic tests, consequently increasing the patient's fear and need for control. Moreover, Walter's wife seemed to have a crucial role in the maintenance of his problem. In fact, despite her best intentions, she was supporting her husband, but further reducing his control over the situation.

As the dialog progressed, the questions became more strategic, the paraphrasing became highly reframed, the language evoked more sensations and finally, the prescriptions became the spontaneous evolution of the dialogue aimed to guide the patient to discover new perceptions that would determine new reactions to the problem (Nardone and Salvini 2007).

"I have two recommendations for you to follow that, I warn you, are not easy to put in place [anticipation technique: the therapist anticipates resistance (a challenge) and makes a statement that prevents the resistance], but let's see what you can do, ok? ... First of all, during the next two weeks, I want you to think that each time you ask for help and receive it, you will simultaneously receive two messages: the first, obvious message is "I love you, help you, and protect you"; while the second message, which is less obvious but stronger and more subtle is, "I help you because you can't make it on your own, because you are incapable". Just as Pessoa (a well-known Portuguese writer who created many alter egos) did, you will bear the wounds of the evaded battles [metaphor that evokes a sensation triggering an emotional effect that leads to the persuasive scope]. Please note that I am not asking you to stop asking for help, because you will not be able to [anticipation technique]. I am only asking you to think that every time you ask for help or reassurance from your wife, and receive it, you contribute to maintaining and worsening your problems. It is as if you pour a special fertilizer on a plant: it makes it grow excessively [metaphor]".

This reframing maneuver driven by repetitive, hypnotic language - and recognized as the "fear of help" — consists of contradictory statements aimed at breaking the mechanism that maintains the problem. In fact, a person who is afraid and who continually asks those around him for reassurance receives reassurance in the moment; he feels safe but, the persistence of this attempted solution-leads to a reduced perception of self-efficacy.

By stating something and immediately after its opposite ("I know that you are not able to stop asking for reassurance, but remember that every time you do, you increase your insecurity rather than decrease it."), the therapist creates aversion towards this behavior without directly telling Walter to do so. The patient is also invited to "avoid avoiding", which is another contradiction.

Then the therapist proceeded by suggesting another therapeutic stratagem named "Heart's Log", which states: "Every day, until we meet again in two weeks, every hour-at 8 am, 9am, 10am, and so on - you will have to count your beats per minute by measuring it from the wrist. To be rigorous, you will have to make three valid measurements, spaced at one-minute intervals. Therefore, every hour of the day, you will have to measure your beats per minute from the wrist. Write down in a notebook the number of the heartbeats you detect, wait for another minute, and check your pulse, registering your heart rate again. Then, wait for one more minute, measure your beats per minute, and write down the result one more time; in this way, a more effective examination of your "crazy" heart rhythm will be obtained by calculating the average heart rate over every time window. This will help us to understand how to keep it controlled".

With this stratagem, the patient is asked to measure his heartbeat every day manually and systematically. By doing so, the need for control of the person is paradoxically reduced by a greater and more systematic control of his symptoms demanded by the professional. 
Heartrate is particularly sensitive to vegetative responses, and the idea of having to measure it is enough to alter its frequency. Repeated measurements are, therefore, necessary to correctly estimate the heart functioning. Moreover, by asking him to do it manually, the therapist leads the patient to properly "meet" his heart, creating a self-regulating effect. Prolonged contact produces familiarity and acceptance, thus altering the modality through which the patient perceives and reacts to his reality. Moreover, by making the patient feel "as if" he is the main protagonist and artefact of the therapeutic change, he will gain responsibility over the achieved and future change.

As expected, Walter reacted to this prescription with excitement, as he felt understood and he could continue to monitor his parameters, and in a more rigorous way than before.

\section{Second Session}

After 2 weeks, the therapist met Walter for the second time and verified the effects of the therapeutic indications on the patient's perceptions and emotions. He stated that avoiding asking for help or reassurance led him to worry less about his problem, and that this happened despite the impossibility for his wife not to intervene. Moreover, the Heart's Log prescription achieved the desired effect, since the rapid heartbeat Walter used to feel decreased in frequency during the last days. Walter was still not sure about the psychological nature of his arrhythmias, but his resistance-once again-was overcome by the use of the strategic dialogue.

As the encounter progressed, the therapist complimented the patient for his effort, invited him to continue to follow the prescriptions, and suggested one more indication.

First, Walter was asked to genuinely thank his wife for her dedication in taking care of him. Then, he was advised to ask her to do something even more challenging in order to help him, that was not to intercede in his support, but "to help him to help himself" by "observing without intervening". The therapist specified that the message must be conveyed in a timely and persuasive manner, since it was aimed to interrupt the attempted solution of his wife without hurting her feelings. Moreover, for the following 2 weeks, Walter was asked to measure his heartbeat per minute from the wrist three times-spaced by 1 -min time, every $3 \mathrm{~h}$.

\section{Third Session}

Walter admitted he was not a "perfect patient": during the days following the last encounter, he proceeded by measuring his heartbeat as suggested, but when he started feeling his heartbeats regularized, he decided to stop the monitoring for a day to see the result, but nothing bad happened. The cardiac frequency apparently returned to normal. "I believe the diagnostic investigations were right. There is nothing wrong with my heartbeat, it was all in my head!" stated Walter while smiling. He experienced a real corrective emotional experience (Alexander and French 1946), that is the vision of a new reality through a process of discovery, which the person thinks he has led and that made the person feelrather than understand-differently about his problem.

Still the systemic attempted solution persisted. In fact, despite the patient being aware of the negative consequences of receiving help and reassurance, it was very difficult for his wife to avoid providing unsolicited help.

Since it was the last possible therapeutic encounter-the therapist proceeded by congratulating him on the results achieved and by strengthening his resources, and motivation to change.

\section{Follow-up Results}

At the 18-month follow-up phone call, Walter reported he was doing well: he continued to occasionally perceive some mild increase of his heart rate that no longer worried him, and that did not lead him to ask for medical examinations other than the routine ones. He also reported working full-time and participating in regular physical activity. Moreover, the support he was still receiving from his wife no longer seemed to interfere with his autonomy and self-efficacy.

\section{Discussion}

This case scenario represents an example of the application of brief strategic therapy in a hospital setting for patients with cardiovascular diseases who also suffer from cardiophobia.

Often, in fact, preoccupation with the heart functioning is allied with a relatively high level of anxiety but minor physical symptoms in both healthy individuals and persons with a heart problem. Reassurance seeking and repeated checking of the heartbeat are important maintaining factors of the problem, as immediate relief is followed by the recurrence of anxiety symptoms (Bartoletti and Nardone 2018). To substitute the dysfunctional attempted solution with more functional ways to cope with the situation-including any unnecessary examinations - traditional forms of psychotherapies make use of reasoning and indicative, descriptive, explanatory, confrontational, and interpretative language (Nardone and Salvini 2007).

On the other hand, from a strategic perspective, change is not demanded by the therapist but is the result of a joint discovery of the therapist and the patient derived through the therapeutic communication.

The first session in brief strategic therapy is the most critical phase of treatment. In fact, as described in this case, 
the initial psychodiagnostic evaluation becomes more than a mere "assessment phase", but a true change-inducing intervention. This is of particular importance in the hospital setting, where working time is limited (Weigl et al. 2009), and the first - and often single - session takes on even greater importance.

After each change or result is achieved, the therapist proceeds to re-orient the therapeutic encounters based on the observed effects. These maneuvers allow the professional to gain operative information on how the problem functions.

Given the absence of clear diagnostic criteria for cardiophobia, this logical operative and strategic way of conducting therapy might help overcome its controversial recognition. In fact, cardiophobia differs from other types of health-related anxiety in that it pertains specifically to the fear of heart-related events and functioning rather than to a variety of bodily sensations - as in panic disorder-or generalized health concerns of individuals with hypochondriasis (American Psychiatric Association 2013). Thus, it seems that cardiophobia does not fit into any of the existing related diagnostic categories. Consequently, patients may receive no diagnosis, or an incorrect diagnosis that may prevent treating the problem (Eifert and Lau 2001).

Among the limitations of this study is the absence of specific measures of heart-focused anxiety, which could have helped to corroborate (or reject) the diagnostic hypothesis that the therapist reached at the end of the treatment based on the patient's responses to evocative communication and suggested prescriptions. Still, no assessment tools in the Italian language are available for this aim, and future research should address this gap.

Moreover, despite the encouraging follow-up outcomes provided by the patient, a more systematic longitudinal assessment of his mood state is lacking-thus preventing reaching reliable conclusions on the effectives of this approach for the treatment of cardiophobia.

Still, reporting of psychological variables showed increased scores in all measures at treatment terminationwith the exception of adherence to medical recommendations. In fact, Walter's self-reported symptoms of anxiety and depression slightly decreased, and his perceived general well-being was one-point higher at treatment termination compared to the baseline measurement. Instead, moderate compliance with treatment was confirmed at the end of the rehabilitation program-probably due to the shared attention of both the patient and his wife on doctors' recommendations that prevent him from full self-management.

However, the ultimate value of a clinical outcome is directly tied to how a given instrument is likely to capture real change, to the degree of change expected, and to whether the contents of a particular measure match the specific goal of the intervention. Indeed, since Walter already performed well on measures of anxiety and depression, and well-being at baseline, then no clinically meaningful changes were expected at treatment termination. Still, participation in treatment, and performance of behavioral prescriptions under very specific conditions were adequate during treatment, and Walter's perceived health-related quality of life increased consistently from the beginning to the end of the rehabilitation program as measured by the EQ-5D-visual analogue scale (VAS). This instrument is commonly used to rate subjective experiences due to its wide score range and high sensitivity, and it is therefore able to capture greater changes in the person's emotional status-even in a short time. Asking the patient how he feels and if he is satisfied with the results achieved with the therapy represents, therefore, the most reliable outcome measure in this case. This conclusion is further supported by the fact that questionnaires were administered at the beginning and at the end of cardiac rehabilitation. Therefore, they fully reflect not only the impact of the brief strategic intervention, but the benefits of the entire program.

\section{Conclusion}

Heart functioning maintains a pivotal role in defining physical and mental health. It is not surprising, therefore, that individuals experiencing cardiac symptoms that might indicate personal danger, but are negative in medical screening, react with symptoms of anxiety that overlap with features of other anxiety-related bodily sensations and events.

This case is an interesting example of how brief strategic therapy can contribute to the development of a new comprehensive conceptual model of how cardiophobia works and maintains itself. This pragmatic form of clinical practice has, in fact, shown preliminary utility for timely recognition and treatment of heart-related anxiety in cardiac rehabilitation. More research is needed to increase theoretical knowledge in the field and to support the efficacy and effectiveness of this approach.

Funding Open access funding provided by Università Cattolica del Sacro Cuore within the CRUI-CARE Agreement.

Open Access This article is licensed under a Creative Commons Attribution 4.0 International License, which permits use, sharing, adaptation, distribution and reproduction in any medium or format, as long as you give appropriate credit to the original author(s) and the source, provide a link to the Creative Commons licence, and indicate if changes were made. The images or other third party material in this article are included in the article's Creative Commons licence, unless indicated otherwise in a credit line to the material. If material is not included in the article's Creative Commons licence and your intended use is not permitted by statutory regulation or exceeds the permitted use, you will need to obtain permission directly from the copyright holder. To view a copy of this licence, visit http://creativecommons.org/licenses/by/4.0/. 


\section{References}

Aikens, J. E., Zvolensky, M. J., \& Eifert, G. H. (2001). Differential fear of cardiopulmonary sensations in emergency room noncardiac chest pain patients. Journal of Behavioral Medicine, 24(2), 155-167. https://doi.org/10.1023/a:1010710614626.

Alexander, F., \& French, T. (1946). Psychoanalytic therapy: Principles and application. New York, NY: Ronald Press.

American Psychiatric Association. (2013). Diagnostic and statistical manual of mental disorders (5th ed.). Arlington, VA: American Psychiatric Association.

Balestroni, G., \& Bertolotti, G. (2012). EuroQol-5D (EQ-5D): An instrument for measuring quality of life. Monaldi Archives for Chest Disease, 78(3), 155-159. https://doi.org/10.4081/monal di.2012.121

Bartoletti, A., \& Nardone, G. (2018). La paura delle malattie. Psicoterapia breve strategica dell'ipocondria. Milan: Ponte alle Grazie.

Bass, C., Wade, C., Hand, D., \& Jackson, G. (1983). Patients with angina with normal and near normal coronary arteries: Clinical and psychosocial state 12 months after angiography. British Medical Journal (Clinical Research Ed.), 287(6404), 1505-1508. https ://doi.org/10.1136/bmj.287.6404.1505.

Beitman, B. D., Kushner, M. G., Basha, I., Lamberti, J., Mukerji, V., \& Bartels, K. (1991). Follow-up status of patients with angiographically normal coronary arteries and panic disorder. Journal of the American Medical Association, 265(12), 1545-1549.

Castelnuovo, G., Manzoni, G. M., Villa, V., Cesa, G. L., Pietrabissa, G., \& Molinari, E. (2011). The STRATOB study: Design of a randomized controlled clinical trial of Cognitive Behavioral Therapy and Brief Strategic Therapy with telecare in patients with obesity and binge-eating disorder referred to residential nutritional rehabilitation. [Comparative Study Randomized Controlled Trial Research Support, Non-U.S. Gov't]. Trials, 12, 114. https://doi. org/10.1186/1745-6215-12-114.

Dupuy, H. J. (1984). The psychological general well-being (PGWB) index. In N. K. Wenger, M. E. Mattson, C. D. Furburg, \& J. Elinson (Eds.), Assessment of quality of life in clinical trials of cardiovascular therapies (pp. 170-183). New York, NY: Le Jacq Publishing.

Eifert, G. H. (1992). Cardiophobia: A paradigmatic behavioural model of heart-focused anxiety and non-anginal chest pain. [Review]. Behaviour Research and Therapy, 30(4), 329-345. https://doi. org/10.1016/0005-7967(92)90045-i.

Eifert, G. H., \& Lau, A. W. (2001). Using behavioral experiments in the treatment of cardiophobia: A case study. Cognitive and Behavioral Practice, 8, 305-316. https://doi.org/10.1016/S1077 -7229(01)80003-1.

Eifert, G. H., Hodson, S. E., Tracey, D. R., Seville, J. L., \& Gunawardane, K. (1996). Heart-focused anxiety, illness beliefs, and behavioral impairment: Comparing healthy heart-anxious patients with cardiac and surgical inpatients. [Research Support, Non-U.S. Gov't]. Journal of Behavioral Medicine, 19(4), 385-399. https:// doi.org/10.1007/BF01904764.

Eifert, G. H., Zvolensky, J. J., \& Lejuez, C. W. (2000). Heart-focused anxiety and chest pain: A conceptual and clinical review. Clinical Psychology: Science and Practice, 7, 403-417. https://doi. org/10.1093/clipsy.7.4.403.

EuroQol Group. (1990). EuroQol—A new facility for the measurement of health-related quality of life. Health Policy, 16(3), 199-208. https://doi.org/10.1016/0168-8510(90)90421-9.

Ginsberg, J. P., Pietrabissa, G., Manzoni, G. M., \& Castelnuovo, G. (2015). Treating the mind to improve the heart: The summon to cardiac psychology. Frontiers in Psychology, 6, 1101. https://doi. org/10.3389/fpsyg.2015.01101.
Grossi, E., Groth, N., Mosconi, P., Cerutti, R., Pace, F., Compare, A., \& Apolone, G. (2006). Development and validation of the short version of the Psychological General Well-Being Index (PGWB-S). [Research Support, Non-U.S. Gov't Validation Studies]. Health and Quality of Life Outcomes, 4, 88. https://doi. org/10.1186/1477-7525-4-88.

Jackson, J. B., Pietrabissa, G., Rossi, A., Manzoni, G. M., \& Castelnuovo, G. (2018). Brief strategic therapy and cognitive behavioral therapy for women with binge eating disorder and comorbid obesity: A randomized clinical trial one-year follow-up. [Randomized Controlled Trial]. Journal of Consulting and Clinical Psychology, 86(8), 688-701. https://doi.org/10.1037/ccp0000313.

Kisely, S. R., Campbell, L. A., Yelland, M. J., \& Paydar, A. (2015). Psychological interventions for symptomatic management of non-specific chest pain in patients with normal coronary anatomy. [Meta-Analysis Research Support, Non-U.S. Gov't Review Systematic Review]. Cochrane Database of Systematic Reviews, 2015(6), CD004101. https://doi.org/10.1002/14651858.CD004 101.pub5.

Kroenke, K., Spitzer, R. L., Williams, J. B., \& Lowe, B. (2009). An ultra-brief screening scale for anxiety and depression: The PHQ-4. [Evaluation Studies Research Support, Non-U.S. Gov't]. Psychosomatics, 50(6), 613-621. https://doi.org/10.1176/appi. psy.50.6.613.

Marker, C. D., Carmin, C. N., \& Ownby, R. L. (2008). Cardiac anxiety in people with and without coronary atherosclerosis. Depression and Anxiety, 25(10), 824-831. https://doi.org/10.1002/da.20348.

Morisky, D. E., Green, L. W., \& Levine, D. M. (1986). Concurrent and predictive validity of a self-reported measure of medication adherence. [Research Support, U.S. Gov't, P.H.S.]. Medical Care, 24(1), 67-74. https://doi.org/10.1097/00005650-19860 1000-00007.

Nardone, G., \& Balbi, E. (2008). Solcare il mare all'insaputa del cielo. Lezioni sul cambiamento terapeutico e le logiche non ordinarie. Milan: Ponte alle Grazie.

Nardone, G., \& Portelli, C. (2005). Knowing through changing: The evolution of brief strategic therapy. Norwalk, CT: Crown House.

Nardone, G., \& Salvini, A. (2007). The strategic dialogue: Rendering the diagnostic interview a real therapeutic intervention. London: Routledge.

Nardone, G., \& Watzlawick, P. (2004). Advanced brief therapy. Northvale, NJ: Aronson.

Ockene, I. S., Shay, M. J., Alpert, J. S., Weiner, B. H., \& Dalen, J. E. (1980). Unexplained chest pain in patients with normal coronary arteriograms: A follow-up study of functional status. The New England Journal of Medicine, 303(22), 1249-1252. https://doi. org/10.1056/NEJM198011273032201.

Pietrabissa, G., Ceccarini, M., Borrello, M., Manzoni, G. M., Titon, A., Nibbio, F., Montano, M., Bertone, G., Gondoni, L., \& Castelnuovo, G. (2015). Enhancing behavioral change with motivational interviewing: A case study in a Cardiac Rehabilitation Unit. Frontiers in Psychology, 6, 298. https://doi.org/10.3389/fpsyg .2015.00298.

Pietrabissa, G., Manzoni, G. M., Gibson, P., Boardman, D., Gori, A., \& Castelnuovo, G. (2016). Brief strategic therapy for obsessivecompulsive disorder: A clinical and research protocol of a onegroup observational study. [Observational Study]. BMJ Open, 6(3), e009118. https://doi.org/10.1136/bmjopen-2015-009118.

Pietrabissa, G., Manzoni, G. M., Rossi, A., \& Castelnuovo, G. (2017). The MOTIV-HEART Study: A prospective, randomized, singleblind pilot study of brief strategic therapy and motivational interviewing among cardiac rehabilitation patients. Frontiers in Psychology, 8, 83. https://doi.org/10.3389/fpsyg.2017.00083.

Rakowska, J. M. (2011). Brief strategic therapy in patients with social phobia with or without personality disorder. Psychotherapy 
Research, 21(4), 462-471. https://doi.org/10.1080/10503 307.2011.581707.

Rakowska, J. M. (2015). Brief strategic therapy in first myocardial infarction patients with increased levels of stress: A randomized clinical trial. [Randomized Controlled Trial]. Anxiety, Stress, and Coping, 28(6), 687-705. https://doi.org/10.1080/10615 806.2015.1004323.

Spinhoven, P., Van der Does, A. J., Van Dijk, E., \& Van Rood, Y. R. (2010). Heart-focused anxiety as a mediating variable in the treatment of noncardiac chest pain by cognitive-behavioral therapy and paroxetine. Journal of Psychosomatic Research, 69(3), 227-235. https://doi.org/10.1016/j.jpsychores.2010.02.005.

Tremblay, M. A., Denis, I., Turcotte, S., Fleet, R. P., Archambault, P., Dionne, C. E., \& Foldes-Busque, G. (2018). Heart-focused anxiety and health care seeking in patients with non-cardiac chest pain: A prospective study. [Research Support, Non-U.S. Gov't]. General Hospital Psychiatry, 50, 83-89. https://doi.org/10.1016/j. genhosppsych.2017.10.007.

Weigl, M., Muller, A., Zupanc, A., \& Angerer, P. (2009). Participant observation of time allocation, direct patient contact and simultaneous activities in hospital physicians. [Evaluation Studies Research Support, Non-U.S. Gov't]. BMC Health Services Research, 9, 110. https://doi.org/10.1186/1472-6963-9-110.

Publisher's Note Springer Nature remains neutral with regard to jurisdictional claims in published maps and institutional affiliations. 